\title{
Charging Interacting Rotating Black Holes in Heterotic String Theory
}

\author{
Alfredo Herrera-Aguilar \\ Theoretical Physics Department, Aristotle University of Thessaloniki \\ 54006 Thessaloniki, Greece \\ and \\ Instituto de Física y Matemáticas, UMSNH \\ Edificio C-3, Ciudad Universitaria, Morelia, Mich. CP 58040 México \\ e-mail: aherrera@auth.gr
}

\begin{abstract}
We present a formulation of the stationary bosonic string sector of the whole toroidally compactified effective field theory of the heterotic string as a double Ernst system which, in the framework of General Relativity describes, in particular, a pair of interacting spinning black holes; however, in the framework of low-energy string theory the double Ernst system can be particularly interpreted as the rotating field configuration of two interacting sources of black hole type coupled to dilaton and Kalb-Ramond fields. We clarify the rotating character of the $B_{t \varphi}$-component of the antisymmetric tensor field of Kalb-Ramond and discuss on its possible torsion nature. We also recall the fact that the double Ernst system possesses a discrete symmetry which is used to relate physically different string vacua. Therefore we apply the normalized Harrison transformation (a charging symmetry which acts on the target space of the low-energy heterotic string theory preserving the asymptotics of the transformed fields and endowing them with multiple electromagnetic charges) on a generic solution of the double Ernst system and compute the generated field configurations for the 4D effective field theory of the heterotic string. This transformation generates the $U(1)^{n}$ vector field content of the whole low-energy heterotic string spectrum and gives rise to a pair of interacting rotating black holes endowed with dilaton, KalbRamond and multiple electromagnetic fields where the charge vectors are orthogonal to each other.
\end{abstract}

PACS numbers: 11.25.Pm, 11.25.Sq, 11.30.Na, 11.25.Mj, 04.20.Jb, 04.50.+h. 


\section{Introduction}

The low energy limit of superstring theories leads to effective field theories of massless fields that are, in fact, supergravity theories coupled to some matter fields. One of the main reasons for constructing classical string solutions to these theories is that their study allows us to address standing problems in quantum gravity since string theory is a promising candidate for a consistent quantum theory of gravity, being free from ultra-violet divergencies that are present in quantum gravity of point-like particles.

With regard to the gravitational field, all the uncharged black hole solutions of general relativity describe as well (up to a good approximation) uncharged black holes in string theory if their mass parameter is large compared to the Plank mass, except in the vicinity of the singularity. This is owing to the fact that the classical equation of motion for the gravitational field in the framework of string theory consists of the Einstein's equation plus Plank correction terms. Thus, as long as the curvature is small compared to the Plank scale, all vacuum solutions of general relativity are approximate solutions of string theory. However, the situation is radically different when one considers solutions of the Einstein-Maxwell (EM) equations. In heterotic string theory, for instance, the dilaton field couples in a non-trivial way to the electromagnetic field, so that every electrovacuum configuration should be accompanied by a non-constant dilaton field. It turns out that the addition of the dilaton field dramatically changes certain properties of black holes [1].

On the other side, it has been observed considerable work in understanding the microscopic origin of the Bekenstein-Hawking entropy of black holes using string theory approaches (for a review see [2]). At the beginning such investigations were mostly carried out for supersymmetric (static extremal or near-extremal) configurations. Later on the study of string and brane physics with no susy became an active field of research, namely, non-BPS branes and brane-antibrane configurations have provided considerable insight into non-perturbative aspects of string/M-theory [3]. In the framework of this background, it is of interest to study more complicated configurations such as interacting black hole sets, both static and rotating, extremal and non-extremal, neutral and charged, and coupled to other matter fields (see, for instance, [4]-[12] and references therein).

The paper is organized as follows. In Sec. 2 we review the matrix Ernst potential (MEP) formulation of the toroidally compactified stationary low-energy heterotic string theory and recall the normalized Harrison symmetry which is a matrix generalization of the Harrison's Bäcklund transformation of the stationary EM theory [13]. We then present the bosonic string truncation of this effective theory as a double Ernst system (in particular, it represents a pair of interacting rotating black holes in the framework of General Relativity) and point out a discrete symmetry of this formulation that relates physically different string vacua in Sec. 3. We further, in Sec. 4, apply the normalized Harrison transformation (NHT) on a generic neutral double Ernst seed solution and recover the $U(1)^{n}$ vector field content of the effective field theory of the heterotic string [14]. We also recall the parametrization of the Ernst potentials that correspond to Kerr black holes in the axially symmetric case. Afterwards, in Sec. 5 we reinterpret the axisymmetric double Ernst system in the framework of string theory as a spinning field configuration consisting of a pair of interacting sources of black hole type coupled to dilaton and Kalb-Ramond fields where the rotating character of the $B_{t \varphi}$-component of the antisymmetric tensor field is explicit. Subsequently, in Sec. 6, we perform a NHT on this field configuration, write down the explicit expression of the generated charged solution and 
study its physical properties. We also analyze some particular cases of the generated solution, qualitatively compare to other previously known field configurations in certain limiting cases and discuss on the physical properties of the considered field configurations and on the further development of this investigation in Sec. 7.

\section{The effective action and matrix Ernst potentials}

We start with the effective action of the heterotic string at tree level

$$
S^{(D)}=\int d^{(D)} x\left|G^{(D)}\right|^{\frac{1}{2}} e^{-\phi^{(D)}}\left(R^{(D)}+\phi_{; M}^{(D)} \phi^{(D) ; M}-\frac{1}{12} H_{M N P}^{(D)} H^{(D) M N P}-\frac{1}{4} F_{M N}^{(D) I} F^{(D) I M N}\right),
$$

where

$$
F_{M N}^{(D) I}=\partial_{M} A_{N}^{(D) I}-\partial_{N} A_{M}^{(D) I}, \quad H_{M N P}^{(D)}=\partial_{M} B_{N P}^{(D)}-\frac{1}{2} A_{M}^{(D) I} F_{N P}^{(D) I}+\text { cycl. perms. of M, N, P. }
$$

Here $G_{M N}^{(D)}$ is the metric, $B_{M N}^{(D)}$ is the anti-symmetric Kalb-Ramond field, $\phi^{(D)}$ is the dilaton and $A_{M}^{(D) I}$ is a set of $U(1)$ vector fields $(I=1,2, \ldots, n)$ and $M, N, P=1,2, \ldots, D$. In the consistent critical case $D=10$ and $n=16$, but we shall leave these parameters arbitrary for the time being and will consider the $D=4$ theory later on. In [15] it was shown that after the compactification of this model on a $D-3=d$-torus, the resulting stationary theory possesses the $S O(d+1, d+1+n)$ symmetry group ( $U$-duality [16]) and describes effective $3 \mathrm{D}$ gravity with the metric tensor

$$
g_{\mu \nu}=e^{-2 \phi}\left(G_{\mu \nu}^{(D)}-G_{m+3, \mu}^{(D)} G_{n+3, \nu}^{(D)} G^{m n}\right)
$$

coupled to the following set of three-fields:

a) scalar fields

$$
G \equiv G_{m n}=G_{m+3, n+3}^{(D)}, \quad B \equiv B_{m n}=B_{m+3, n+3}^{(D)}, \quad A \equiv A_{m}^{I}=A_{m+3}^{(D) I}, \quad \phi=\phi^{(D)}-\frac{1}{2} \ln |\operatorname{det} G|,
$$

b)antisymmetric tensor field

$$
B_{\mu \nu}=B_{\mu \nu}^{(D)}-4 B_{m n} A_{\mu}^{m} A_{\nu}^{n}-2\left(A_{\mu}^{m} A_{\nu}^{m+d}-A_{\nu}^{m} A_{\mu}^{m+d}\right),
$$

c) vector fields $A_{\mu}^{(a)}=\left(\left(A_{1}\right)_{\mu}^{m},\left(A_{2}\right)_{\mu}^{m+d},\left(A_{3}\right)_{\mu}^{2 d+I}\right)$

$$
\left(A_{1}\right)_{\mu}^{m}=\frac{1}{2} G^{m n} G_{n+3, \mu}^{(D)},\left(A_{3}\right)_{\mu}^{I+2 d}=-\frac{1}{2} A_{\mu}^{(D) I}+A_{n}^{I} A_{\mu}^{n},\left(A_{2}\right)_{\mu}^{m+d}=\frac{1}{2} B_{m+3, \mu}^{(D)}-B_{m n} A_{\mu}^{n}+\frac{1}{2} A_{m}^{I} A_{\mu}^{I+2 d},
$$

where the subscripts $m, n=1,2, \ldots, d ; \mu, \nu=1,2,3$; and $a=1, \ldots, 2 d+n$. In this paper we set $B_{\mu \nu}=0$ to remove the effective cosmological constant from our consideration.

All vector fields in three dimensions can be dualized on-shell with the aid of the pseudoscalar fields $u, v$ and $s$ as follows:

$$
\begin{aligned}
& \nabla \times \overrightarrow{A_{1}}=\frac{1}{2} e^{2 \phi} G^{-1}\left(\nabla u+\left(B+\frac{1}{2} A A^{T}\right) \nabla v+A \nabla s\right) \\
& \nabla \times \overrightarrow{A_{3}}=\frac{1}{2} e^{2 \phi}\left(\nabla s+A^{T} \nabla v\right)+A^{T} \nabla \times \overrightarrow{A_{1}} \\
& \nabla \times \overrightarrow{A_{2}}=\frac{1}{2} e^{2 \phi} G \nabla v-\left(B+\frac{1}{2} A A^{T}\right) \nabla \times \overrightarrow{A_{1}}+A \nabla \times \overrightarrow{A_{3}} .
\end{aligned}
$$


Thus, the effective stationary theory describes gravity $g_{\mu \nu}$ coupled to the scalars $G, B, A, \phi$ and pseudoscalars $u, v, s$. These matter fields can be arranged in the following pair of matrix Ernst potentials:

$$
\mathcal{X}=\left(\begin{array}{cc}
-e^{-2 \phi}+v^{T} X v+v^{T} A s+\frac{1}{2} s^{T} s & v^{T} X-u^{T} \\
X v+u+A s & X
\end{array}\right), \quad \mathcal{A}=\left(\begin{array}{c}
s^{T}+v^{T} A \\
A
\end{array}\right),
$$

where $X=G+B+\frac{1}{2} A A^{T}$. These matrices have dimensions $(d+1) \times(d+1)$ and $(d+1) \times n$, respectively, and their components have the following physical meaning: the relevant information about the gravitational field is contained in the matrix potential $X$ through the matrix $G$, whereas its rotational character is encoded in the dualized variable $u ; X$ also parameterizes the antisymmetric Kalb-Ramond tensor field $B$, whereas its multidimensional components are dualized through $v$; the 3D dilaton is $\phi$, and $A$ and $s$ stand for electric and magnetic potentials, respectively. In terms of the MEP the effective stationary theory adopts the form

$$
{ }^{3} S=\int d^{3} x|g|^{\frac{1}{2}}\left\{-R+\operatorname{Tr}\left[\frac{1}{4}\left(\nabla \mathcal{X}-\nabla \mathcal{A} \mathcal{A}^{T}\right) \mathcal{G}^{-1}\left(\nabla \mathcal{X}^{T}-\mathcal{A} \nabla \mathcal{A}^{T}\right) \mathcal{G}^{-1}+\frac{1}{2} \nabla \mathcal{A}^{T} \mathcal{G}^{-1} \nabla \mathcal{A}\right]\right\},
$$

where $\mathcal{X}=\mathcal{G}+\mathcal{B}+\frac{1}{2} \mathcal{A} \mathcal{A}^{T}$, then $\mathcal{G}=\frac{1}{2}\left(\mathcal{X}+\mathcal{X}^{T}-\mathcal{A} \mathcal{A}^{T}\right)$ and

$$
\mathcal{G}=\left(\begin{array}{cc}
-e^{-2 \phi}+v^{T} G v & v^{T} G \\
G v & G
\end{array}\right), \quad \mathcal{B}=\left(\begin{array}{cc}
0 & v^{T} B-u^{T} \\
B v+u & B
\end{array}\right) .
$$

In [17] it was shown that there exist a map between the stationary actions of the low-energy heterotic string and Einstein-Maxwell theories. The map reads

$$
\mathcal{X} \longleftrightarrow-E, \quad \mathcal{A} \longleftrightarrow F
$$

and also involves an interchange of the matrix transposition operation with the complex conjugation one. Here $E$ and $F$ are the complex Ernst potentials (gravitational and electromagnetic, respectively) of the stationary EM theory [18]. This map allows us to extrapolate the results obtained in the EM theory to the heterotic string one using the MEP formulation.

\subsection{The normalized Harrison transformation}

In [17] it was shown that the total symmetry group $S O(d+1, d+1+n) /[S O(d+1) \times S O(d+n+1)]$ of the stationary low-energy effective field theory of the heterotic string can be decomposed into scaling, electromagnetic rotation, gauge and non-linear (Ehlers and Harrison) matrix transformations in terms of the MEP, just as this takes place in the stationary EM theory. Further, following the work of Kinnersley [19] for the stationary EM theory, the symmetries of the stationary action (8) were classified according to their charging properties in [20]. It turns out that only the generalized Ehlers and Harrison transformations act in a non-trivial way on a given seed space-time since they introduce physical charges into the generated solution (by applying a scaling transformation on a seed field configuration we just make it larger or smaller without adding any charge and, in this sense, without changing its physical properties, the electromagnetic rotation leaves unchanged a charged configuration; finally, the gauge symmetries of the MEP do not alter a given seed solution). 
At this point we would like to mention that the NHT constitutes a matrix generalization of the charging symmetry of Bäcklund type introduced by Harrison in the framework of the EM theory [13]. In that context, by applying the Harrison transformation on a neutral seed solution one introduces a complex parameter which endows with electric and/or magnetic charges the generated field configuration. On the other side, by applying the normalized Ehlers transformation on a given seed solution, one does not introduce charges of electromagnetic nature since starting with a neutral configuration one ends again with a neutral solution (within the context of General Relativity, it introduces the NUT parameter).

Thus, in the framework of the effective field theory of the heterotic string, under the NHT we can construct charged string vacua from neutral ones with the same asymptotical values of the fields, just as this takes place in the framework of General Relativity where we obtain the Kerr-Newman black hole from the Kerr one under such non-linear symmetry. However, in the low-energy string theory context, the NHT introduces $(d+1) \times n$ real parameters through a matrix $\lambda$ and generates a field configuration endowed with $(d+1) \times n$ electromagnetic charges starting from a neutral seed solution. In the concrete case of the critical effective field theory of the heterotic string, when $D=10$ and $n=16$, it generates 128 electromagnetic charges.

In this respect, the non-linear NHT selects from the total $S O(d+1, d+1+n) /[S O(d+1) \times$ $S O(d+n+1)]$ symmetry group of the stationary effective field theory of the heterotic string, the symmetries that endow a given seed field configuration with electromagnetic charges. Another important issue of this method concerns its applicability to stationary systems only, thus, it cannot be implemented to effective field theories depending on more than three space-time dimensions.

In this respect we would like to point out that the solution generating technique implemented in the present work is quite different from the method based in orthogonal transformations that has been applied in [15] and [21], for instance. However, it seems that the result of applying both techniques is quite similar and in some cases, the same. It is interesting to study the relationship between both solution generating methods.

Thus, the matrix transformation

$$
\begin{aligned}
& \mathcal{A} \rightarrow\left(1+\frac{1}{2} \Sigma \lambda \lambda^{T}\right)\left(1-\mathcal{A}_{0} \lambda^{T}+\frac{1}{2} \mathcal{X}_{0} \lambda \lambda^{T}\right)^{-1}\left(A_{0}-\mathcal{X}_{0} \lambda\right)+\Sigma \lambda \\
& \mathcal{X} \rightarrow\left(1+\frac{1}{2} \Sigma \lambda \lambda^{T}\right)\left(1-\mathcal{A}_{0} \lambda^{T}+\frac{1}{2} \mathcal{X}_{0} \lambda \lambda^{T}\right)^{-1}\left[\mathcal{X}_{0}+\left(\mathcal{A}_{0}-\frac{1}{2} \mathcal{X}_{0} \lambda\right) \lambda^{T} \Sigma\right]+\frac{1}{2} \Sigma \lambda \lambda^{T} \Sigma,
\end{aligned}
$$

where $\Sigma=\operatorname{diag}(-1,-1 ; 1,1, \ldots, 1)$ and $\lambda$ is an arbitrary constant $(d+1) \times n$-matrix parameter, generates charged string solutions (with non-zero potential $\mathcal{A}$ ) from neutral ones if we start from the seed potentials

$$
\mathcal{X}_{0} \neq 0 \quad \text { and } \quad \mathcal{A}_{0}=0 \text {. }
$$

This solution generation procedure allows us to generate the $U(1)^{n}$ electromagnetic spectrum

of the effective field theory of the heterotic string starting with just field spectrum of the low-energy bosonic string theory.

\section{Bosonic string truncation vs double Ernst system}

Let us consider just the low-energy bosonic string sector of the whole effective stationary heterotic string theory. Thus, we must set to zero all the $U(1)$ field strengths which correspond 
to the winding modes of the reduced theory; in terms of the MEP this is equivalent to dropping the matrix $\mathcal{A}$ in the action (8)

$$
{ }^{3} S=\int d^{3} x|g|^{\frac{1}{2}}\left\{-R+\frac{1}{4} \operatorname{Tr}\left[\nabla \mathcal{X} \mathcal{G}^{-1} \nabla \mathcal{X}^{T} \mathcal{G}^{-1}\right]\right\}=\int d^{3} x|g|^{\frac{1}{2}}\left\{-R+\frac{1}{4} \operatorname{Tr}\left(J^{\mathcal{X}} J^{\mathcal{X}^{T}}\right)\right\}
$$

where now $\mathcal{X}=\mathcal{G}+\mathcal{B}, \mathcal{G}=\frac{1}{2}\left(\mathcal{X}+\mathcal{X}^{T}\right)$ and $J^{\mathcal{X}}=\nabla \mathcal{X} \mathcal{G}^{-1}$.

There are several physically different theories (and truncations) that can be expressed by the action (11), and hence admit a double Ernst formulation (see, for instance, [5]-[6]); among them we find the $D=4$ low-energy bosonic string theory, the bosonic sector of $D=4, \mathcal{N}=4$ supergravity, the $D=5$ Einstein-Kalb-Ramond effective model, where the dilaton field is set to zero, etc. In these cases $\mathcal{G}$ and $\mathcal{B}$ are $2 \times 2-$ matrices and can be parameterized in the following form

$$
\mathcal{G}=\frac{p_{1}}{p_{2}}\left(\begin{array}{cc}
1 & q_{2} \\
q_{2} & p_{2}^{2}+q_{2}^{2}
\end{array}\right), \quad \mathcal{B}=q_{1}\left(\begin{array}{cc}
0 & -1 \\
1 & 0
\end{array}\right)
$$

Thus, the action of the matter fields takes the form

$$
{ }^{3} S_{m}=\frac{1}{2} \int d^{3} x|g|^{\frac{1}{2}}\left\{p_{1}^{-2}\left[\left(\nabla p_{1}\right)^{2}+\left(\nabla q_{1}\right)^{2}\right]+p_{2}^{-2}\left[\left(\nabla p_{2}\right)^{2}+\left(\nabla q_{2}\right)^{2}\right]\right\},
$$

which allows us to introduce two independent to each other Ernst-like potentials

$$
\epsilon_{1}=p_{1}+i q_{1}, \quad \epsilon_{2}=p_{2}+i q_{2} .
$$

In terms of these field variables, the action of the system can be rewritten as a double Ernst system in the Kähler form [22]:

$$
{ }^{3} S=\int d^{3} x|g|^{\frac{1}{2}}\left\{-{ }^{3} R+2\left(J^{\epsilon_{1}} J^{\bar{\epsilon}_{1}}+J^{\epsilon_{2}} J^{\bar{\epsilon}_{2}}\right)\right\},
$$

where $J^{\epsilon_{k}}=\nabla \epsilon_{k}\left(\epsilon_{k}+\bar{\epsilon}_{k}\right)^{-1}$ and $k=1,2$. In the framework of the stationary General Relativity each complex Ernst potential describes, in general, a spinning field configuration and, in particular, an axially symmetric rotating black hole (or spherically symmetric static black hole when the potential is real). Thus, the action (15) represents the linear superposition of two rotating black holes among other configurations.

A mathematically equivalent, but physically different $2 \times 2-$ matrix representation arises from (13) by making use of the discrete symmetry $p_{1} \longleftrightarrow p_{2}, \quad q_{1} \longleftrightarrow q_{2}$. This fact allows us to define the new matrices $\mathcal{G}^{\prime}$ and $\mathcal{B}^{\prime}$, and hence the new matrix potential $\mathcal{X}^{\prime}=\mathcal{G}^{\prime}+\mathcal{B}^{\prime}$, and to write down the action that corresponds to the primed quantities:

$$
{ }^{3} S=\int d^{3} x|g|^{\frac{1}{2}}\left\{-R+\frac{1}{4} \operatorname{Tr}\left(J^{\mathcal{X}^{\prime}} J^{\mathcal{X}^{\prime T}}\right)\right\}=\int d^{3} x|g|^{\frac{1}{2}}\left\{-R+2\left(J^{\epsilon_{1}^{\prime}} J^{\bar{\epsilon}_{1}^{\prime}}+J^{\epsilon_{2}^{\prime}} J^{\bar{\epsilon}_{2}^{\prime}}\right)\right\},
$$

where similarly $J^{\mathcal{X}^{\prime}}=\nabla \mathcal{X}^{\prime} \mathcal{G}^{\prime-1}, \quad J^{\prime}=\nabla \epsilon_{k}^{\prime}\left(\epsilon_{k}^{\prime}+\bar{\epsilon}_{k}^{\prime}\right)^{-1}, \epsilon_{1}^{\prime}=p_{2}+i q_{2}$ and $\epsilon_{2}^{\prime}=p_{1}+i q_{1}$.

In terms of the MEP the above-mentioned discrete transformation reads $\mathcal{X} \longleftrightarrow \mathcal{X}^{\prime}$; thus, the matrices $\mathcal{G}^{\prime}$ and $\mathcal{B}^{\prime}$ must be interpreted as new Kaluza-Klein and Kalb-Ramond fields. This symmetry mixes the gravitational and matter degrees of freedom of the theories. It works like the Bonnor transformation of the Einstein-Maxwell theory [23], but in the bosonic string realm. It can be used to generate new solutions starting from seed solutions as pure Kaluza-Klein or Kaluza-Klein-Dilaton string vacua. 


\section{Charging double Ernst solutions in string theory}

For concreteness, in this Sec. we shall consider the truncated $D=4$ action of the effective field theory of the bosonic string with a non-null timelike Killing vector; thus, the vectors fields of the effective field theory of the heterotic string encoded in the MEP $\mathcal{A}$ vanish and the stationary low-energy action is effectively described by (11) with $\mathcal{G}$ and $\mathcal{B}$ defined by (12). Subsequently, we will generate the electromagnetic sector $\left(U(1)^{n}\right.$ vector field content) of the low-energy heterotic string theory via the NHT and, finally, will impose axial symmetry in order to consider the MEP corresponding to a couple of interacting Kerr black holes.

The seed MEP that correspond to the neutral stationary double Ernst system are

$$
\mathcal{X}_{0}=\left(\begin{array}{cc}
\frac{p_{1}}{p_{2}} & \frac{p_{1} q_{2}-q_{1} p_{2}}{p_{2}} \\
\frac{p_{1} q_{2}+q_{1} p_{2}}{p_{2}} & \frac{p_{1}}{p_{2}}\left(p_{2}^{2}+q_{2}^{2}\right)
\end{array}\right), \quad \mathcal{A}_{0}=0
$$

For the simplest case the charge matrix $\lambda$ that parameterizes the NHT is conformed by two vector rows as follows

$$
\lambda=\left(\begin{array}{cccc}
\lambda_{11} & \lambda_{12} & \ldots & \lambda_{1 n} \\
\lambda_{21} & \lambda_{22} & \ldots & \lambda_{2 n}
\end{array}\right)
$$

where $n \geq 2$ for consistency; the parameters $\lambda_{1 i}$ and $\lambda_{2 i}$ can be interpreted as the electromagnetic charges of the generated field configuration. When $n=6$ the generated field spectrum corresponds to the bosonic sector of $\mathcal{N}=4, D=4$ supergravity; however, here we shall leave it arbitrary for the sake of generality. After applying the NHT on the generic double Ernst seed solution (17) with the matrix $\lambda(18)$, the transformed MEP read

$$
\begin{aligned}
& \mathcal{X}_{11}=\frac{1}{\Xi}\left[\left(4+\Lambda^{2}\left|\epsilon_{2}\right|^{2}\right) R e \epsilon_{1}+2\left(\lambda_{1 i}^{2}+\lambda_{2 i}^{2}\left|\epsilon_{1}\right|^{2}\right) R e \epsilon_{2}-4 \lambda_{1 i} \lambda_{2 i} R e \epsilon_{2} \operatorname{Im} \epsilon_{1}\right], \\
& \mathcal{X}_{12}=\frac{1}{\Xi}\left\{\Gamma_{-}\left(\operatorname{Re}_{1} I m \epsilon_{2}-\operatorname{Re}_{2} \operatorname{Im} \epsilon_{1}\right)+2 \lambda_{1 i} \lambda_{2 i}\left[\left(1-\left|\epsilon_{2}\right|^{2}\right) \operatorname{Re}_{1}+\left(1-\left|\epsilon_{1}\right|^{2}\right) R e \epsilon_{2}\right]\right\}, \\
& \mathcal{X}_{21}=\frac{1}{\Xi}\left\{\Gamma_{+}\left(\operatorname{Re}_{1} I m \epsilon_{2}+\operatorname{Re}_{2} \operatorname{Im} \epsilon_{1}\right)+2 \lambda_{1 i} \lambda_{2 i}\left[\left(1-\left|\epsilon_{1}\right|^{2}\right) R e \epsilon_{2}-\left(1-\left|\epsilon_{2}\right|^{2}\right) R e \epsilon_{1}\right]\right\}, \\
& \mathcal{X}_{22}=\frac{1}{\Xi}\left[\left(\Lambda^{2}+4\left|\epsilon_{2}\right|^{2}\right) R e \epsilon_{1}+2\left(\lambda_{2 i}^{2}+\lambda_{1 i}^{2}\left|\epsilon_{1}\right|^{2}\right) R e \epsilon_{2}+4 \lambda_{1 i} \lambda_{2 i} \operatorname{Re} \epsilon_{2} \operatorname{Im} \epsilon_{1}\right], \\
& \mathcal{A}_{1 j}=\frac{-2}{\Xi}\left\{\left[\left(2+\lambda_{2 i}^{2}\left|\epsilon_{2}\right|^{2}\right) R e \epsilon_{1}+\left(2+\lambda_{2 i}^{2}\left|\epsilon_{1}\right|^{2}\right) R e \epsilon_{2}+\lambda_{1 i} \lambda_{2 i}\left(R e \epsilon_{1} I m \epsilon_{2}-R e \epsilon_{2} I m \epsilon_{1}\right)\right] \lambda_{1 j}+\right. \\
& \left.\left[\left(2-\lambda_{1 i}^{2}\right)\left(R e \epsilon_{1} I m \epsilon_{2}-R e \epsilon_{2} I m \epsilon_{1}\right)-\lambda_{1 i} \lambda_{2 i}\left(\left|\epsilon_{2}\right|^{2} R e \epsilon_{1}+\left|\epsilon_{1}\right|^{2} R e \epsilon_{2}\right)\right] \lambda_{2 j}\right\}, \\
& \mathcal{A}_{2 j}=\frac{-2}{\Xi}\left\{\left[\left(2-\lambda_{2 i}^{2}\right)\left(\operatorname{Re\epsilon }_{1} \operatorname{Im} \epsilon_{2}+\operatorname{Re}_{2} \operatorname{Im} \epsilon_{1}\right)-\lambda_{1 i} \lambda_{2 i}\left(R e \epsilon_{1}+\left|\epsilon_{1}\right|^{2} \operatorname{Re}_{2}\right)\right] \lambda_{1 j}+\right.
\end{aligned}
$$




$$
\left.\left[\left(\lambda_{1 i}^{2}+2\left|\epsilon_{2}\right|^{2}\right) R e \epsilon_{1}+\left(2+\lambda_{1 i}^{2}\left|\epsilon_{1}\right|^{2}\right) R e \epsilon_{2}+\lambda_{1 i} \lambda_{2 i}\left(R e \epsilon_{1} I m \epsilon_{2}+R e \epsilon_{2} I m \epsilon_{1}\right)\right] \lambda_{2 j}\right\},
$$

where $\Xi=2\left(\lambda_{1 i}^{2}+\lambda_{2 i}^{2}\left|\epsilon_{2}\right|^{2}\right) R e \epsilon_{1}+\left(4+\Lambda^{2}\left|\epsilon_{1}\right|^{2}\right) R e \epsilon_{2}+4 \lambda_{1 i} \lambda_{2 i} R e \epsilon_{1} I m \epsilon_{2}, \Gamma_{-}=4-2 \lambda_{1 i}^{2}+2 \lambda_{2 i}^{2}-\Lambda^{2}$, $\Gamma_{+}=4+2 \lambda_{1 i}^{2}-2 \lambda_{2 i}^{2}-\Lambda^{2}$ and $\Lambda^{2}=\lambda_{1 i}^{2} \lambda_{2 j}^{2}-\left(\lambda_{1 i} \lambda_{2 i}\right)^{2}$. It is straightforward to check that the generated MEP preserve the asymptotic trivial values of the seed MEP $\mathcal{X}_{0}$ and $\mathcal{A}_{0}$.

It remains just to consider concrete seed solutions to the double Ernst system, to substitute the expressions for $\epsilon_{1}$ and $\epsilon_{2}$ in the transformed MEP formulae, to compute the original fields of the theory and to give an interpretation of the generated families of solutions.

\subsection{Axisymmetric case and interacting Kerr black holes}

Since most of the physically meaningful solutions of General Relativity that can be used as seed solutions in our approach are axisymmetric, we shall impose axial symmetry and write the line element in the Lewis-Papapetrou form using canonical Weyl coordinates

$$
d s^{2}=\left(d x^{m}+2\left(A_{1}\right)_{\varphi}^{m} d \varphi\right)^{T} G_{m n}\left(d x^{n}+2\left(A_{1}\right)_{\varphi}^{n} d \varphi\right)+e^{2 \phi}\left[e^{2 \gamma}\left(d \rho^{2}+d z^{2}\right)+\rho^{2} d \varphi^{2}\right],
$$

where $\gamma, \phi, G_{m n}$ and $\left(A_{1}\right)_{\varphi}^{m}$ are $\varphi$-independent. A solution of our axisymmetric system can be constructed using the solutions of the double vacuum Einstein equations written in the Ernst form in terms of $\epsilon_{k}$ and $\gamma^{\epsilon_{k}}$

$$
\begin{aligned}
\nabla\left(\rho J^{\epsilon_{k}}\right) & =\rho J^{\epsilon_{k}}\left(J^{\epsilon_{k}}-J^{\bar{\epsilon}_{k}}\right) \\
\partial_{z} \gamma^{\epsilon_{k}} & =\rho\left[\left(J^{\epsilon_{k}}\right)_{z}\left(J^{\bar{\epsilon}_{k}}\right)_{\rho}+\left(J^{\bar{\epsilon}_{k}}\right)_{z}\left(J^{\epsilon_{k}}\right)_{\rho}\right] \\
\partial_{\rho} \gamma^{\epsilon_{k}} & =\rho\left[\left|\left(J^{\epsilon_{k}}\right)_{\rho}\right|^{2}-\left|\left(J^{\epsilon_{k}}\right)_{z}\right|^{2}\right]
\end{aligned}
$$

if one identifies the function $\gamma$, that accounts for the general relativistic interaction between de black holes, in the following way $\gamma \equiv \gamma^{\epsilon_{1}}+\gamma^{\epsilon_{2}}$ because of the linear character that possesses the superposition of the sources in the action (15).

Let us consider the double Kerr solution as the seed one. The Ernst potentials corresponding to two Kerr black holes with sources in different points of the symmetry axis are:

$$
\epsilon_{k}=1-\frac{2 m_{k}}{r_{k}+i a_{k} \cos \theta_{k}}
$$

where $m_{k}$ and $a_{k}$ are constant parameters which define the masses and rotations of the sources of the Kerr field configurations. Weyl and Boyer-Lindquist coordinates are related through

$$
\rho=\sqrt{\left(r_{k}-m_{k}\right)^{2}-\sigma_{k}^{2}} \sin \theta_{k}, \quad z=z_{k}+\left(r_{k}-m_{k}\right) \cos \theta_{k},
$$

where the sources are located at $z_{k}$ and $\sigma_{k}^{2}=m_{k}^{2}-a_{k}^{2}$. Thus, for the function $\gamma^{\epsilon_{k}}$ we have $e^{2 \gamma^{\epsilon} k}=P_{k} / Q_{k}$, where $P_{k}=\Delta_{k}-a_{k}^{2} \sin ^{2} \theta_{k}, \quad Q_{k}=\Delta_{k}+\sigma_{k}^{2} \sin ^{2} \theta_{k}$ and $\Delta_{k}=r_{k}^{2}-2 m_{k} r_{k}+a_{k}^{2}$.

\section{Seed solutions of $D=4$ heterotic string theory}

Let us consider some particular solutions for the stationary action of the $D=4$ effective field theory of the bosonic string. In this case the three-dimensional field configurations of the low-energy theory are parameterized in terms of the double Ernst system as follows

$$
G_{t t}=\frac{R e \epsilon_{1}}{R e \epsilon_{2}}\left|\epsilon_{2}\right|^{2}, \quad B \equiv 0, \quad e^{-2 \phi}=-\frac{R e \epsilon_{1} R e \epsilon_{2}}{\left|\epsilon_{2}\right|^{2}}, \quad u=I m \epsilon_{1}, \quad v=\frac{I m \epsilon_{2}}{\left|\epsilon_{2}\right|^{2}},
$$


In particular, if we consider the case when the Ernst potentials correspond to a double Kerr black hole, the $4 \mathrm{D}$ field configuration in the string frame reads

$$
\begin{gathered}
d s_{4}^{2}=G_{t t}\left(d t+\omega_{\varphi} d \varphi\right)^{2}+\frac{e^{2 \phi^{(4)}}}{\left|G_{t t}\right|}\left[e^{2 \gamma}\left(d \rho^{2}+d z^{2}\right)+\rho^{2} d \varphi^{2}\right], \\
G_{t t}=-\frac{P_{1} R_{2}}{\tilde{r}_{1} P_{2}}, \quad \omega_{\varphi} \equiv 2\left(A_{1}\right)_{\varphi}=\frac{2 m_{1} \alpha_{1} r_{1} \sin ^{2} \theta_{1}}{P_{1}}, \quad e^{2 \gamma}=\frac{P_{1} P_{2}}{Q_{1} Q_{2}}, \\
e^{\phi^{(4)}}=\frac{R_{2}}{P_{2}}, \quad B_{t \varphi} \equiv 2\left(A_{2}\right)_{\varphi}=\frac{2 m_{2} \alpha_{2} \sin ^{2} \theta_{2}\left(r_{2}-2 m_{2}\right)}{P_{2}},
\end{gathered}
$$

where $R_{k}=\left(r_{k}-2 m_{k}\right)^{2}+\alpha_{k}^{2} \cos ^{2} \theta_{k}$ and $\tilde{r}_{k}=r_{k}^{2}+\alpha_{k}^{2} \cos ^{2} \theta_{k}$, and describe the axisymmetric rotating field configuration of two interacting sources $m_{1}$ and $m_{2}$ of black hole type located at $z_{1}$ and $z_{2}$ respectively, and endowed with dilaton and Kalb-Ramond fields. It is worth noting that the $G_{t t}$-component of the metric tensor defines the horizon of each gravitational source, however, the quantity $\omega_{\varphi}$ parameterizes only the rotation of the source $m_{1}$ since it does not depend on the parameter $\alpha_{2}$. Interestingly, the Kalb-Ramond field possesses a rotational character (compare it to $\omega_{\varphi}$ ), depends just on the parameter $\alpha_{2}$ and has no Coulomb term in its asymptotical decomposition. Thus, the rotating character of the gravitational field generated by the source $m_{2}$, in the framework of General Relativity, effectively translates into a 'rotating' antisymmetric background in the framework of low-energy string theory. This fact could be related to the torsion nature of the $B_{t \varphi}$-component of the antisymmetric tensor field of KalbRamond, however, these interpretation deserves a separate investigation and will be pursued elsewhere. Finally, let us point out that the $4 \mathrm{D}$ dilaton field is located at $z_{2}$. The explicit expressions for $\left(A_{1}\right)_{\varphi}$ and $\left(A_{2}\right)_{\varphi}$ have been computed using the dualization formulae (6) with the aid of the following coordinate transformation

$$
\begin{aligned}
2 r_{i} & =\sqrt{\rho^{2}+\left(z-z_{i}+\sqrt{m_{i}^{2}-\alpha_{i}^{2}}\right)^{2}}+\sqrt{\rho^{2}+\left(z-z_{i}-\sqrt{m_{i}^{2}-\alpha_{i}^{2}}\right)^{2}}+2 m_{i}, \\
2 \cos \theta_{i} & =\left(\sqrt{\rho^{2}+\left(z-z_{i}+\sqrt{m_{i}^{2}-\alpha_{i}^{2}}\right)^{2}}-\sqrt{\rho^{2}+\left(z-z_{i}-\sqrt{m_{i}^{2}-\alpha_{i}^{2}}\right)^{2}}\right) / \sqrt{m_{i}^{2}-\alpha_{i}^{2}} .
\end{aligned}
$$

Since at spatial infinity $\left.G_{t t}\right|_{\infty} \longrightarrow-1$, either $\epsilon_{1}$ or $\epsilon_{2}$ must adopt the asymptotic value -1 ; for concreteness we have chosen $\left.\epsilon_{1}\right|_{\infty} \longrightarrow-1$. Looking at the asymptotical behaviour of the $G_{t t}$ component of the gravitational tensor we get

$$
\left.G_{t t}\right|_{\infty} \sim-1+\frac{2\left(m_{1}+m_{2}\right)}{\rho}
$$

as expected since the double black hole is generated by the masses $m_{1}$ and $m_{2}$; on the other side, for the dilaton field we asymptotically have the following relation

$$
\left.\varphi\right|_{\infty} \sim \frac{\left(m_{1}-m_{2}\right)}{\rho} \equiv \frac{d}{\rho},
$$


which defines its charge. We see that this three-dimensional dilaton charge effectively vanishes in the case when $m_{1}=m_{2}=m$.

Due to the discrete symmetry $\epsilon_{1} \leftrightarrow \epsilon_{2}$, there is an alternative parameterization of the seed fields

$$
G_{t t}^{\prime}=\frac{R e \epsilon_{2}}{R e \epsilon_{1}}\left|\epsilon_{1}\right|^{2}, \quad B^{\prime} \equiv 0, \quad e^{-2 \phi^{\prime}}=\frac{-R e \epsilon_{1} R e \epsilon_{2}}{\left|\epsilon_{1}\right|^{2}}, \quad v^{\prime}=\frac{I m \epsilon_{1}}{\left|\epsilon_{1}\right|^{2}}, \quad u^{\prime}=I m \epsilon_{2},
$$

where we observe certain duality between the antisymmetric Kalb-Ramond field and the nondiagonal term $G_{t \varphi}$, responsible for the rotation of the gravitational field. This relationship was already pointed out in [6] and [24] and can be seen by switching off one of the potentials, say $\epsilon_{2}=1$; thus, the resulting configuration corresponds to a single $4 \mathrm{D}$ rotating black hole with vanishing dilaton and Kalb-Ramond fields. Under the discrete symmetry mentioned above, this solution is mapped into a static field configuration endowed with non-trivial dilaton and Kalb-Ramond fields. It it interesting to study in more detail the string vacua that are related via this discrete duality and establish its physical implications (for explicit examples see [25]).

\section{Charged field configurations in heterotic string theory}

After applying the NHT on the seed solution (29) we get the following three-dimensional fields:

$$
\begin{gathered}
G \equiv G_{t t}=\mathcal{X}_{22}-\frac{1}{2} \mathcal{A}_{2 j}^{2}, \quad B \equiv 0, \quad A=\mathcal{A}_{2 j}, \quad v=\frac{1}{2 G}\left(\mathcal{X}_{12}+\mathcal{X}_{21}-\mathcal{A}_{1 j} \mathcal{A}_{2 j}\right) \\
u=v \mathcal{X}_{22}-\mathcal{X}_{12}, \quad s^{T}=\mathcal{A}_{1 j}-v \mathcal{A}_{2 j}, \quad e^{-2 \phi}=\frac{1}{2}\left[v\left(\mathcal{X}_{12}+\mathcal{X}_{21}\right)+\mathcal{A}_{i j} s\right]-\mathcal{X}_{11} .
\end{gathered}
$$

where the appearance of the electromagnetic potential is obvious. Similar relations hold for the primed field system that arises under the interchange $\epsilon_{1} \longleftrightarrow \epsilon_{2}$.

With the aid of the dualization relations (6) we get explicit expressions for the non-trivial components of the vector fields

$$
\begin{gathered}
\omega_{\varphi} \equiv 2\left(A_{1}\right)_{\varphi}=\left[-4 \chi_{11}+2 \lambda_{1 i}^{2} \chi_{21}+\left(\lambda_{1 i}^{2} \lambda_{2 j}^{2}-\left(\lambda_{1 i} \lambda_{2 i}\right)^{2}\right) \chi_{12}-2 \lambda_{2 i}^{2} \chi_{22}+4 \lambda_{1 i} \lambda_{2 i} \chi_{23}\right] / D Q \\
B_{t \varphi} \equiv 2\left(A_{2}\right)_{\varphi}=\left[-2 \lambda_{2 i}^{2} \chi_{11}+\left(\lambda_{1 i}^{2} \lambda_{2 j}^{2}-\left(\lambda_{1 i} \lambda_{2 i}\right)^{2}\right) \chi_{21}+2 \lambda_{1 i}^{2} \chi_{12}-4 \chi_{22}-4 \lambda_{1 i} \lambda_{2 i} \chi_{13}\right] / D Q \\
\left(A_{3}\right)_{\varphi}^{I}=(D Q)^{-2}\left\{\lambda _ { 1 i } \lambda _ { 2 i } \left[D Q\left(\chi_{12}+\chi_{21}\right)-\left(4-2 \lambda_{2 i}^{2}\right) \chi_{22}+\left(\lambda_{1 i}^{2} \lambda_{2 j}^{2}-2 \lambda_{1 i}^{2}-\left(\lambda_{1 i} \lambda_{2 i}\right)^{2}\right) \chi_{21}-\right.\right. \\
\left.\left.4 \lambda_{1 i} \lambda_{2 i} \chi_{13}\right]+2\left[\left(1-\lambda_{1 i}^{2}\right)\left(2-\lambda_{2 j}^{2}\right)^{2}-\left(1-\lambda_{2 i}^{2}\right)\left(\lambda_{1 j} \lambda_{2 j}\right)^{2}\right]\left(\chi_{13}-\chi_{23}\right)\right\} \lambda_{1 I}+ \\
(D Q)^{-2}\left\{2 D Q\left(\chi_{11}+\chi_{22}\right)-D Q \lambda_{1 i}^{2}\left(\chi_{12}+2 \chi_{21}\right)+2 D Q \lambda_{1 i} \lambda_{2 i}\left(\chi_{13}-\chi_{23}\right)-\right. \\
\left.2 \lambda_{1 i} \lambda_{2 i}\left[\lambda_{1 j} \lambda_{2 j}\left(\chi_{21}+\chi_{22}\right)+\left(4-\lambda_{1 j}^{2}-\lambda_{2 j}^{2}\right) \chi_{13}+\left(\lambda_{2 j}^{2}-\lambda_{1 j}^{2}\right) \chi_{23}\right]\right\} \lambda_{2 I},
\end{gathered}
$$


where $I=1,2, \ldots n$ and the functions $\chi_{k l}(l=1,2,3 ;)$ read

$\chi_{k 1}=\frac{\alpha_{k} m_{k} r_{k} \sin ^{2} \theta_{k}}{P_{k}}, \quad \chi_{k 2}=\frac{\alpha_{k} m_{k}\left(r_{k}-2 m_{k}\right) \sin ^{2} \theta_{k}}{P_{k}}, \quad \chi_{k 3}=\frac{m_{k}\left(r_{k}^{2}-2 m_{k} r_{k}+\alpha_{k}^{2}\right) \cos \theta_{k}}{P_{k}}$,

and $D Q=4-2 \lambda_{1 i}^{2}-2 \lambda_{2 i}^{2}+\lambda_{1 i}^{2} \lambda_{2 j}^{2}-\left(\lambda_{1 i} \lambda_{2 i}\right)^{2}$. It is worth noticing that even though the generated MEP are asymptotically flat, the $\chi_{k 3}$ functions do not vanish at spatial infinity (they involve the so-called NUT parameters of the gravitational field and the Dirac strings of the magnetic components of the vector fields). Thus, in order to get asymptotically flat gravitational field configurations, which include charged black holes, we must get rid of the terms proportional to $\chi_{k 3}$. This can be done by imposing the orthogonality condition on the pair of charge vectors $\lambda_{1 i}$ and $\lambda_{2 i}$ :

$$
\lambda_{1 i} \lambda_{2 i}=0
$$

however, even with this restriction the magnetic components of the gauge fields $\left(A_{3}\right)_{\varphi}^{I}$ still present the Dirac string singularity; in order to remove it we need to normalize the charge vector $\lambda_{1 i}$ as follows $\lambda_{1 i}^{2}=1$, but we shall leave it unnormalized since these singularities could correspond to Dirac strings of gravitating monopole solutions. Thus, after imposing (36), the transformed metric preserves its form (30) with the following fields in the string frame

$$
\begin{aligned}
& G_{t t}=-\frac{\left(2-\lambda_{2 i}^{2}\right)^{2} P_{1} P_{2}\left[4 R_{2} \tilde{r}_{1}+\lambda_{1 j}^{4} R_{1} \tilde{r}_{2}-4 \lambda_{1 j}^{2}\left(P_{1} P_{2}-4 m_{1} m_{2} \alpha_{1} \alpha_{2} \cos \theta_{1} \cos \theta_{2}\right)\right]}{\left[P_{2}\left(4 \tilde{r}_{1}+\lambda_{1 j}^{2} \lambda_{2 i}^{2} R_{1}\right)-2 P_{1}\left(\lambda_{1 j}^{2} \tilde{r}_{2}+\lambda_{2 i}^{2} R_{2}\right)\right]^{2}}, \\
& e^{\phi^{(4)}}=\frac{\left(2-\lambda_{2 i}^{2}\right)\left[4 R_{2} \tilde{r}_{1}+\lambda_{1 j}^{4} R_{1} \tilde{r}_{2}-4 \lambda_{1 j}^{2}\left(P_{1} P_{2}-4 m_{1} m_{2} \alpha_{1} \alpha_{2} \cos \theta_{1} \cos \theta_{2}\right)\right]}{\left(2-\lambda_{1 j}^{2}\right)\left[-2 P_{1}\left(\lambda_{1 j}^{2} \tilde{r}_{2}+\lambda_{2 i}^{2} R_{2}\right)+P_{2}\left(4 \tilde{r}_{1}+\lambda_{1 j}^{2} \lambda_{2 i}^{2} R_{1}\right)\right]}, \\
& \omega_{\varphi} \equiv 2\left(A_{1}\right)_{\varphi}=2\left(4 \chi_{11}-2 \lambda_{1 j}^{2} \chi_{21}-\lambda_{1 j}^{2} \lambda_{2 i}^{2} \chi_{12}+2 \lambda_{2 i}^{2} \chi_{22}\right) /\left[\left(2-\lambda_{1 j}^{2}\right)\left(2-\lambda_{2 i}^{2}\right)\right] \\
& B_{t \varphi}^{(4)} \equiv 2\left(A_{2}\right)_{\varphi}=2\left(2 \lambda_{2 i}^{2} \chi_{11}-\lambda_{1 j}^{2} \lambda_{2 i}^{2} \chi_{21}-2 \lambda_{1 j}^{2} \chi_{12}+4 \chi_{22}\right) /\left[\left(2-\lambda_{1 j}^{2}\right)\left(2-\lambda_{2 i}^{2}\right)\right] \\
& A^{I}=\frac{4\left(2-\lambda_{2 i}^{2}\right)^{2}\left(m_{2} \alpha_{2} \cos \theta_{2} P_{1}+m_{1} \alpha_{1} \cos \theta_{1} P_{2}\right) \lambda_{1 I}}{\left[P_{2}\left(4 \tilde{r}_{1}+\lambda_{1 j}^{2} \lambda_{2 i}^{2} R_{1}\right)-2 P_{1}\left(\lambda_{1 j}^{2} \tilde{r}_{2}+\lambda_{2 i}^{2} R_{2}\right)\right]}+ \\
& \frac{2\left[P_{1}\left(\lambda_{1 j}^{2} \tilde{r}_{2}+2 R_{2}\right)-P_{2}\left(2 \tilde{r}_{1}+\lambda_{1 j}^{2} R_{1}\right)\right] \lambda_{2 I}}{\left[P_{2}\left(4 \tilde{r}_{1}+\lambda_{1 j}^{2} \lambda_{2 i}^{2} R_{1}\right)-2 P_{1}\left(\lambda_{1 j}^{2} \tilde{r}_{2}+\lambda_{2 i}^{2} R_{2}\right)\right]}, \\
& \left(A_{3}\right)_{\varphi}^{I}=4\left(1-\lambda_{1 j}^{2}\right)\left(\chi_{23}-\chi_{13}\right) \lambda_{1 I} /\left(2-\lambda_{1 j}^{2}\right)^{2}- \\
& 2\left[2\left(\chi_{11}+\chi_{22}\right)-\lambda_{1 j}^{2}\left(\chi_{12}+2 \chi_{21}\right)\right] \lambda_{2 I} /\left[\left(2-\lambda_{1 j}^{2}\right)\left(2-\lambda_{2 i}^{2}\right)\right],
\end{aligned}
$$


where $e^{2 \gamma}$ remains the same. It is straightforward to check that when one sets to zero the charge vectors $\lambda_{k i}$ one recovers the seed field configuration (30)-(31).

By switching to the Einstein frame, where most of the calculations for gravitational and thermodynamical analysis are performed, we obtain

$$
d s_{E}^{2}=e^{-\phi^{(4)}} d s_{s t}^{2}=-f\left(d t-\omega_{\varphi}\right)^{2}+f^{-1}\left[e^{2 \gamma}\left(d \rho^{2}+d z^{2}\right)+\rho^{2} d \varphi^{2}\right],
$$

where the component of the metric tensor $f$ reads

$$
f=\frac{\left(2-\lambda_{1 j}^{2}\right)\left(2-\lambda_{2 i}^{2}\right) P_{1} P_{2}}{\left[P_{2}\left(4 \tilde{r}_{1}+\lambda_{1 j}^{2} \lambda_{2 i}^{2} R_{1}\right)-2 P_{1}\left(\lambda_{1 j}^{2} \tilde{r}_{2}+\lambda_{2 i}^{2} R_{2}\right)\right]}
$$

and has the following asymptotic behaviour

$$
\left.f\right|_{\infty} \sim 1-\frac{2\left(M_{1}+M_{2}\right)}{r}, \quad \text { with } \quad M_{1} \equiv \frac{\left(4-\lambda_{1 j}^{2} \lambda_{2 i}^{2}\right) m_{1}}{\left(2-\lambda_{1 j}^{2}\right)\left(2-\lambda_{2 i}^{2}\right)}, \quad M_{2} \equiv \frac{2\left(\lambda_{2 j}^{2}-\lambda_{1 j}^{2}\right) m_{2}}{\left(2-\lambda_{1 j}^{2}\right)\left(2-\lambda_{2 i}^{2}\right)}
$$

where $M_{1}$ and $M_{2}$ are the effective masses of the sources located at $z_{1}$ and $z_{2}$, respectively. A novel feature of this configuration is that the function $\omega_{\varphi} \equiv 2\left(A_{1}\right)_{\varphi}$ now contains information about the spinning character of the gravitational field generated by both sources since it has the following asymptotic behaviour

$$
\left.\omega_{\varphi}\right|_{\infty} \sim \frac{2 J_{1} \sin ^{2} \theta_{1}}{r}+\frac{2 J_{2} \sin ^{2} \theta_{2}}{r} \quad \text { with } \quad J_{1}=M_{1} \alpha_{1}, \quad J_{2}=M_{2} \alpha_{2}
$$

where $J_{1}$ and $J_{2}$ define the angular momenta generated by the spinning sources $M_{1}$ and $M_{2}$. This is a quite interesting effect because of the dependence of $\omega_{\varphi}$ on both rotational parameters $\alpha_{1}$ and $\alpha_{2}$. In fact, the $G_{t \varphi}$-component of the seed field configuration did not depend on $a_{2}$, however, the NHT induces a rotation in the charge space which takes place in such a way that the source located at $z_{2}$ gets as well an effective gravitational angular momentum (apart from the rotating field described by the $B_{t \varphi}$-component of the Kalb-Ramond tensor field).

On the other hand, we analyze the asymptotic behaviour of the three-dimensional electric and magnetic potentials $A^{I}$ and $s_{I}^{T}$

$$
\left.A^{I}\right|_{\infty} \sim \frac{Q_{2 I}}{r},\left.\quad s_{I}^{T}\right|_{\infty} \sim \frac{\mu_{1 I}}{r}, \quad \text { with } \quad Q_{2 I} \equiv \frac{-4\left(m_{1}+m_{2}\right) \lambda_{2 I}}{\left(2-\lambda_{2 i}^{2}\right)}, \quad \mu_{1 I} \equiv \frac{4\left(m_{2}-m_{1}\right) \lambda_{1 I}}{\left(2-\lambda_{1 i}^{2}\right)} \text {, }
$$

where $Q_{2 I}$ and $\mu_{1 I}$ determine the electric and magnetic charges of the gauge fields, respectively.

\section{Conclusion and Discussion}

We have presented a double Ernst formulation of the stationary bosonic string theory with some classes of exact solutions which can be interpreted as a rotating field configuration generated by two interacting sources of black hole type endowed with dilaton and Kalb-Ramond fields. We clarify the rotating character of the $B_{t \varphi}^{(4)}$-component of the antisymmetric tensor field of Kalb-Ramond; this fact could be related to the torsion nature of the antisymmetric background generated by the Kalb-Ramond tensor field. Thus, string theory could predict the necessary 
presence of torsion when studying gravitational field configurations coupled or not to other matter fields. This line of investigation deserves more attention and we hope to develop it in the near future (for a review on this issue see, for example [26]).

By starting with a given field configuration of the stationary effective field theory of the bosonic string, we obtained a new configuration with the full field spectrum of the truncated low-energy $D=4$ heterotic string theory via a straightforward generation of the electromagnetic sector of the latter theory with the aid of the non-linear matrix transformation of Harrison type. We imposed as well the necessary condition on the charge vectors in order to get asymptotically flat 4D gravitational field configurations. Thus, in this way we have generated a field configuration which consists of a pair of charged interacting rotating black holes coupled to dilaton and Kalb-Ramond fields and endowed with multiple electromagnetic charges (when $n=6$ the field spectrum of the considered theory also describes the bosonic sector of $D=\mathcal{N}=4$ supergravity).

In the framework of General Relativity the complete class of solutions of the EM theory which describes an axisymmetric field configuration consisting of a pair of black holes located on the axis with arbitrary mass, charge and rotating parameters was constructed in [4] with an original method implemented by Sibgatullin [27]. Due to its cumbersome form, it is really difficult to extract particular solutions from it. We would like to point out the main difference between the exact solution presented here and that of [4] (which gave rise to further investigations [9]-[12]). The point is that the interaction between the black holes is different in both cases, namely, in our configuration, the parameterization of the MEP $\mathcal{X}$ which leads to the double Ernst potential formulation of the bosonic string theory fixes the linear superposition of the sources (at the level of the Lagrangian), leading in turn to the interaction given by $e^{2 \gamma}=e^{2\left(\gamma_{\epsilon_{1}}+\gamma_{\epsilon_{2}}\right)}$ which is different from the interaction considered in [4] in the framework of the Sibgatullin's method (see also [28]), namely, the derivation of the solution involves the choice of a plausible form of the Ernst potentials on the symmetry axis $(\rho=0, z)$ and therefore extends these expressions to the whole $(\rho, z)$ plane; the Ernst potential on the axis chosen in [4] reads

$$
\mathcal{E}(\rho=0, z) \equiv e(z)=\frac{\left(z+z_{1}-m_{1}-i a_{1}\right)\left(z+z_{2}-m_{2}-i a_{2}\right)}{\left(z+z_{1}+m_{1}-i a_{1}\right)\left(z+z_{2}+m_{2}-i a_{2}\right)}
$$

whereas in the framework of this method our choice corresponds to the potential

$$
\epsilon_{k}(\rho=0, z) \equiv e^{\prime}(z)=\frac{\left(z-z_{1}-m_{1}+i a_{1}\right)}{\left(z-z_{1}+m_{1}+i a_{1}\right)}+\frac{\left(z-z_{2}-m_{2}+i a_{2}\right)}{\left(z-z_{2}+m_{2}+i a_{2}\right)} ;
$$

this difference leads to distinct functions $e^{2 \gamma}$ and, thus, to different interactions.

It should be pointed out that the integral equation method of Sibgatullin is more powerful than the just algebraic method of nonlinear matrix transformations of Bäcklund type. However, in the framework of string theory, one could choose more general seed Ernst potentials than the (27) ones and get therefore the same interaction generated by the Sibgatullin method. This issue deserves more attention and will be studied as well in a further work.

When certain parameters are set to zero, we recover some previously studied configurations, however, one needs to perform a great amount of algebraic computations in order to extract explicit expressions for these solutions. We shall just point out that when the Kalb-Ramond field and rotational parameters vanish and we have just one electric charge at $z_{1}$ and $z_{2}$, we reproduce a field configuration obtained by [7] and a solution quite similar to the black dilohe considered in [8]-[9]. 
We would like to emphasize as well that in the framework of General Relativity the problem of equilibrium between two rotating black holes has been intensively studied (see, for example, [29] and references therein) and practically solved [10]-[11].

It is interesting to analyze other physical properties of the constructed solution such as energy, equilibrium, thermodynamical behaviour, dual configurations, etc. One can extract as well gravitating monopole-antimonopole systems and uplift the constructed solutions to 10 dimensions in order to reinterpret them as brane-antibrane configurations. Some of these issues are under current investigation.

\section{Acknowledgements}

The author acknowledges useful discussions with Deborah Aguilera and Oleg Kechkin and thanks IMATE-UNAM and CINVESTAV for provided library facilities while part of this investigation was in progress. He is really grateful to S. Kousidou for encouraging him during the performance of this article.

The author would like to express his gratitude to the Theoretical Physics Department of the Aristotle University of Thessaloniki and, specially, to Prof. J.E. Paschalis for fruitful discussions and for providing a stimulating atmosphere while the final version of this work was completed. He also acknowledges a grant for postdoctoral studies provided by the Greek Government. This research was supported by grants CIC-UMSNH-4.18 and CONACYT-J34245-E and F42064.

\section{References}

[1] D. Garfinkle, G. Horowitz and A. Strominger, Phys. Rev. D43 (1991) 3140; Erratum-ibid. D45 (1992) 3888.

[2] D. Youm, Phys. Rept. 316 (1999) 1; A.W. Peet, "TASI lectures on black holes in string theory", in 'Boulder 1999, Strings, branes and gravity' (1999) 353; hep-th/0008241 and references therein.

[3] A. Sen, "Non-BPS states and branes in string theory", in 'Cargese 1999, Progress in string theory and M-theory' (1999) 187; hep-th/9904207.

[4] V.S. Manko, J. Martin and E. Ruiz, J. Math. Phys. 35 (1994) 6644.

[5] D.V. Galtsov and O.V. Kechkin, Phys. Lett. B361 (1995) 52.

[6] A. Herrera-Aguilar and O. Kechkin, Mod. Phys. Lett. A12 (1997) 1629.

[7] M.S. Costa and M.J. Perry, Nucl. Phys. B591 (2000) 469.

[8] R. Emparan, Phys. Rev. D61 (2000) 104009.

[9] R. Emparan and E. Teo, Nucl. Phys. B610 (2001) 190.

[10] V.S. Manko, E. Ruiz and J.D. Sanabria-Gómez, Class. Quant. Grav. 17 (2000) 3881.

[11] N. Bretón, V.S. Manko and J. Aguilar-Sánchez, Class. Quant. Grav. 16 (1999) 3725. 
[12] Y.C. Liang and E. Teo, Phys. Rev. D64 (2001) 024019.

[13] B.K. Harrison, J. Math. Phys. 9 (1968) 1744; Phys. Rev. Lett. 41 (1978) 119.

[14] A. Herrera-Aguilar, Rev. Mex. Fís. 49S2 (2003) 141.

[15] J. Maharana and J.H. Schwarz, Nucl. Phys. B390 (1993) 3; A. Sen, Nucl. Phys. B434 (1995) 179.

[16] C.M. Hull and P.K. Towsend, Nucl. Phys. B438 (1995) 109.

[17] A. Herrera and O. Kechkin, Int. J. Mod. Phys. A13 (1998) 393; A14 (1999) 1345.

[18] F.J. Ernst, Phys. Rev. 167 (1968) 1175.

[19] W. Kinnersley, J. Math. Phys. 21 (1980) 2231.

[20] A. Herrera-Aguilar and O. Kechkin, Phys. Rev. D59 (1999) 124006.

[21] S.F. Hassan and A. Sen, Nucl. Phys. B375 (1992) 103.

[22] P.O. Mazur, Act. Phys. Pol. B14 (1983) 219.

[23] W. Bonnor, Z. Phys. 190 (1966) 444; E. Fischer, J. Math. Phys. 20 (1979) 2547.

[24] A. Herrera-Aguilar and O. Kechkin, Mod. Phys. Lett. A16 (2001) 29.

[25] A. Herrera-Aguilar and M. Nowakowski, Class. Quant. Grav. 21 (2004) 1015.

[26] R.T. Hammond, Rep. Prog. Phys. 21 (2002) 599.

[27] N.R. Sibgatullin, Oscillations and Waves in Strong Gravitational and Electromagnetic Fields (Nauka, Moscow, 1984; English Translation: Springer-Verlag, Berlin, 1991).

[28] A. Chamorro, V.S. Manko and N.R. Sibgatullin, Lect. Notes Phys. 423 (1993) 119.

[29] A. Tomimatsu and M. Kihara, Prog. Theor. Phys. 67 (1982) 349; 67 (1982) 1406; A. Tomimatsu, Prog. Theor. Phys. 71 (1984) 409. 